\title{
LOCAL QUANTITIES FOR THE 1D HUBBARD MODEL IN THE COMPOSITE OPERATOR METHOD
}

\author{
A. Avella, F. Mancini, M. M. Sánchez, D. Villani \\ Università degli Studi di Salerno - Unità INFM di Salerno \\ Dipartimento di Scienze Fisiche "E. R. Caianiello" \\ 84081 Baronissi (SA), Italy, \\ F. D. Buzatu \\ National Institute for Physics and Nuclear Engineering \\ Dept. of Theoretical Physics, 76900 Măgurele, Bucharest, Romania
}

(Received March 9, 1998)

\begin{abstract}
The discovery of new materials with properties dominated by strong correlations among electrons has opened the problem of new appropriate calculation schemes. In the case of 1D models the Bethe ansatz provides an exact evaluation of many relevant physical quantities, but not a complete framework. By way of developing new methods appropriate to strongly correlated systems, we study the 1D Hubbard model by means of the Composite Operator Method. We investigate various local quantities. A comparison with exact results and other analytical approaches show a reasonable agreement and determine the applicability range of our approximate scheme.
\end{abstract}

Key words: 1D Hubbard model, composite operator method.

PACS number(s): 71.10.+x, 71.27.+a

\section{INTRODUCTION}

Electron correlations in narrow energy bands play a key role in the description of many new materials that present anomalous behaviors. The Hubbard model is the simplest Hamiltonian containing the essence of strong correlation and its one-dimensional (1D) version can give us the basic understanding of this field. In addition, in nature we find real quasi-1D systems like TTF-TCNQ and $\mathrm{KCP}$ salts [1] and the $\mathrm{Cu}-\mathrm{O}$ chains of the high- $T_{c}$ cuprates that seem to show features whose comprehension requires an intensive study of the $1 \mathrm{D}$ models. Moreover, since Anderson proposed that the high- $T_{c}$ superconductors should be considered as Luttinger liquids [2], the 1D Hubbard model, as paradigm of these systems, has been widely studied.

As it is well known, the Bethe ansatz solution for the 1D Hubbard model provides an exact evaluation of some local quantities as the energy of the ground state, the double occupancy, the energy gap and so on [3]. Nevertheless, this ansatz cannot be thought of as a complete framework for the 1D Hubbard model, since if we want to evaluate more relevant physical properties of the system, i.e. the response functions, we must address other approaches. On the one hand we have numerical methods as the quantum Monte Carlo and Lanczos [4]; on the other we can turn to many analytical approaches [5-7]. However, the small size of the clusters and the impossibility of working at very low temperatures are inherent to all numerical techniques; whereas, not all the available analytical methods give satisfactory results.

In this paper we want to present an alternative analytical approach to the study of the 1D Hubbard model, that does not present the inconveniences mentioned above. This method, called the Composite Operator Method
(COM) [8], is based on the idea that the long-lived excitations present in the system could be better described by some auxiliary fermionic fields (whose properties are self-consistently determined by the dynamics) than by the standard electronic ones. Moreover, the use of such a non-standard basis in the determination of the Green's function leads to the appearance of some free parameters not directly connected to the elements of the Green's function itself. In COM, these parameters are fixed selfconsistently by imposing some symmetry requirements, like the Pauli principle and the particle-hole symmetry [8]. This procedure permits to recover these symmetries, usually badly violated by other approaches, and thus to properly describe strongly correlated systems, where the interplay between self-consistency and symmetry requirement could be the only practicable way to face the problem. This line of thinking has given its fruits when applied to many systems [8]. The results obtained for the thermodynamic and magnetic properties are in good agreement with the numerical data. Some anomalous behaviors, experimentally observed in high- $T_{c}$ cuprate superconductors, have also been explained successfully [8]. The reliability of COM on the evaluation of the relevant response functions of the $1 \mathrm{D}$ Hubbard model can be deduced by a comparison with the Bethe ansatz results on the local quantities. Also, the comparison with other analytical approaches [5-7] will allow to establish the relevance and reliability of the method.

\section{METHOD}

The 1D Hubbard model is described by the following Hamiltonian: 


$$
H=\sum_{i, j} t_{i j} c^{\dagger}(i) c(j)+U \sum_{i} n_{\uparrow}(i) n_{\downarrow}(i),
$$

where $c^{\dagger}(i)=\left(c_{\uparrow}^{\dagger}(i), c_{\downarrow}^{\dagger}(i)\right)$ is the electron operator on site $i$ in the spinor notation, $n_{\sigma}(i)$ is the charge-density operator for the spin $\sigma$ and $n(i)$ is the total chargedensity operator. In the hopping matrix

$$
\begin{aligned}
& t_{i j}=-4 t \alpha_{i j}=-4 t \frac{1}{N} \sum_{k} e^{i k(i-j)} \alpha(k), \\
& \alpha(k)=\frac{1}{2} \cos k
\end{aligned}
$$

only the terms up to the nearest neighbors have been considered; $\alpha_{i j}$ is the projector on the nearest neighbors. The $U$ parameter is the onsite Coulomb repulsion.

The 2D Hubbard model has been previously analyzed by means of $\mathrm{COM}$ in the static approximation where finite life-time effects are neglected using the Hubbard doublet as a basic field [8]. The single-particle retarded thermal Green's function is a function of the external parameters $t, U, n$ (filling) and $T$ (temperature) and three internal parameters $\mu, \Delta$ and $p$. The latter have been determined using a system of self-consistent equations which come from the existing relations with the elements of the Green's function and from the requirement of the Pauli principle at the level of matrix elements. In the case of the 1D Hubbard model the same scheme of calculations can be applied. The resulting formulas differ from the ones of the $2 \mathrm{D}$ model only in the explicit expression for the $\alpha$ projector.

In the framework of COM, the Fourier transform of the single-particle retarded thermal Green's function may be written as:

$$
S(k, \omega)=\sum_{i=1}^{2} \frac{\sigma^{i}(k)}{\omega-E_{i}(k)},
$$

where the spectral moments $\sigma^{1,2}(k)$ are given by

$$
\begin{aligned}
& \sigma_{11}^{i}(k)=\frac{I_{1}\left(2 Q(k)+(-)^{i+1} \Delta \Sigma(k)\right)}{4 Q(k)}, \\
& \sigma_{12}^{i}(k)=\sigma_{21}^{i}(k)=(-)^{i+1} \frac{m(k)}{2 Q(k)}, \\
& \sigma_{22}^{i}(k)=\frac{I_{2}\left(2 Q(k)+(-)^{i} \Delta \Sigma(k)\right)}{4 Q(k)}
\end{aligned}
$$

with

$$
I_{1}=1-\frac{n}{2}, \quad I_{2}=\frac{n}{2},
$$

$$
\begin{aligned}
& Q(k)=\frac{1}{2} \sqrt{\left(U-\frac{m(k)}{I_{11} I_{22}}\right)^{2}+2 n U \frac{m(k)}{I_{11} I_{22}}}, \\
& R(k)=\frac{1}{2}(U-2 \mu-8 t \alpha(k))-\frac{1}{2} \frac{m(k)}{I_{11} I_{22}} \\
& \Delta \Sigma(k)=(1-n) \frac{m(k)}{I_{11} I_{22}}-U, \\
& m(k)=4 t\left(\Delta+\alpha(k)\left(p-I_{2}\right)\right)
\end{aligned}
$$

and $\mu$ the chemical potential.

The energy bands $E_{1,2}(k)$ have the following expression:

$$
E_{i}(k)=R(k)+(-)^{i+1} Q(k) .
$$

The internal energy has been calculated following two different schemes. One determination comes directly by taking the thermal average of the Hamiltonian $\left(E_{H}\right)$. The other one is obtained by introducing the Helmhotz free energy per site

$$
F=E-T S,
$$

where $S$ is the entropy per site. From thermodynamics we have

$$
S=-\frac{\partial F}{\partial T}, \mu=\frac{\partial F}{\partial n} \Rightarrow \frac{\partial S}{\partial n}=-\frac{\partial \mu}{\partial T} .
$$

Then, it is straightforward to get the expression

$$
E_{T}=\int_{0}^{n}\left(\mu(T, \tilde{n})-T \frac{\partial \mu}{\partial T}(T, \tilde{n})\right) d \tilde{n} .
$$

\section{RESULTS}

We have studied the paramagnetic solution of the model and found two solutions of the coupled selfconsistent equations that will be called henceforth COM 1 and COM 2. They differ principally in the sign of the $p$ parameter ( $p<0$ for COM 1 and $p>0$ for COM 2) which drives the overall shape of the bands (see Eqs. 12 and 13). The first solution has very strong antiferromagnetic correlations with respect to the second one. As can be seen in Fig. 1a, COM 1 presents a typical antiferromagnetic band pattern, i.e. a very narrow bandwidth of the order $J=\frac{4 t^{2}}{U}$, the first excitation at $k= \pm \frac{\pi}{2}$ and a quasi-halved Brillouin zone. COM 2 shows a bandwidth of the order $8 t$, the first hole excitation at $k= \pm \pi$ and the first electron excitation at $k=0$. In Fig. 1b, COM 1 shows a gap in the excitation spectrum which is almost equal to $U$. This is consistent with the Bethe ansatz results which present a gap for any value of $U$. Nevertheless, the magnitude of the gap increases too much in the 
weak coupling regime, but reaches the Bethe ansatz rate for the values of $U \sim 4$. On the contrary, COM 2 is characterized by the critical value $U_{c} \sim 6.6$, above which the gap opens.
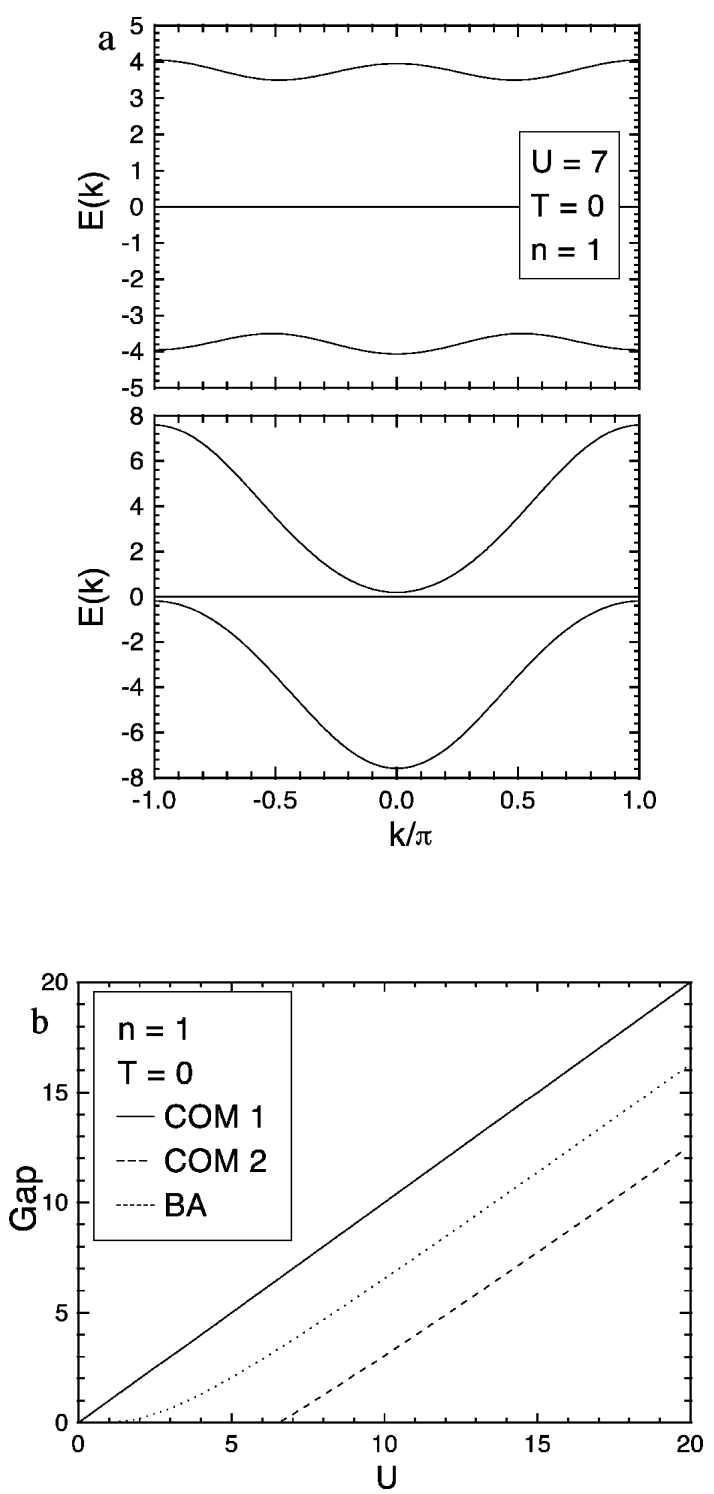

Fig. 1. a) Energy bands for COM 1 (upper panel) and COM 2 (lower panel); b) Energy gap for COM 1, COM 2 and BA (Bethe ansatz).

The energy of the ground state for the two solutions has been calculated both thermodynamically and through the average of the Hamiltonian. In the following figures, we will present only the lowest determination of the energy in the chosen range of external parameters. We present the results for the ground-state energy of the system obtained with our method ( $E_{H}^{\mathrm{COM} 1}$ ), Bethe ansatz and other analytical approaches [5-7] (see Fig. 2a). The excellent agreement between our result and Bethe ansatz is correlated to the opening of the gap men- tioned above. We can also see that both the Ladder [7] and the Gutzwiller approximations [6] go to zero at the finite value of $U$, whereas COM, together with the Renormalization Group [5], has the right asymptotic behavior. Nevertheless the latter approach fails completely in the free limit $(U=0)$. The self-consistent Ladder approximation [7] has a good behavior for all regime of couplings, but gives a finite ground state energy for infinite $U$. The double occupancy and kinetic energy in COM 1 for the same value of the external parameters is also in qualitative agreement with the Bethe ansatz results.
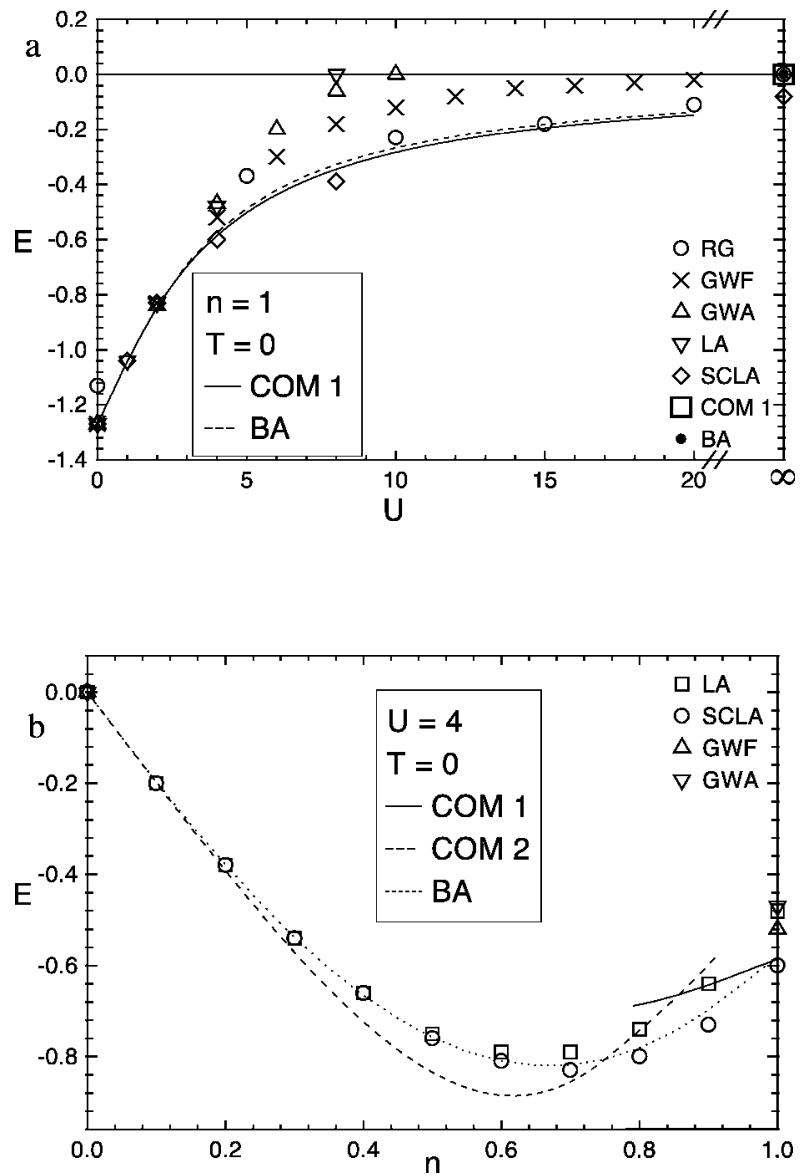

Fig. 2. a) Ground state energy for $\operatorname{COM} 1\left(E_{H}\right), \mathrm{BA}, \mathrm{RG}$ (Renormalization Group), GWF (Gutzwiller wave function), GWA (Gutzwiller approximation), LA (Ladder approximation) and SCLA (Self consistent LA); b) As in (a) for COM 1 $\left(E_{H}\right)$ COM $2\left(E_{T}\right)$, BA, GWF, GWA and LA.

Fig. 2b shows the ground-state energy away from half filling for an intermediate value of the coupling. Following the minimum energy criterium mentioned above, we plot COM 1 near half-filling and COM 2 otherwise. This fact can be interpreted as a transition, within our paramagnetic solution, from a ground state characterized by very weak antiferromagnetic correlations away from half filling to another one where these correlations are very 
strong near half filling. The agreement between our results and Bethe ansatz is excellent very near half filling where, as it is known, the system is in an antiferromagnetic ground state. On the contrary, the Ladder approximation [7], the Gutzwiller wave function and Gutzwiller method [6] deviate from Bethe ansatz at half-filling. The self-consistent ladder approximation [7] probes excellently at any doping but deviates slightly at half-filling.

\section{CONCLUSIONS}

The search for new schemes of approximation has been renewed by the discovery of new materials dominated by strong electronic correlations. The Bethe ansatz solution of the 1D Hubbard model does not provide a complete framework for calculations since it does not allow the evaluation of many response functions of the system. We have used the Composite Operator Method to study some local quantities and compare our results to Bethe ansatz as well as to other analytical approaches. The proposed method has a very good agreement with the exact result at half-filling for any value of the coupling; for intermediate value of both doping and coupling the deviation is not more than $10 \%$. In spite of considering a paramagnetic solution, at half-filling we found a ground state with strong antiferromagnetic correlations; this state shows a gap for any value of the coupling in agreement with the Bethe ansatz solution, although the increasing rate is larger in our case. From the present results, our method seems to be a reasonable framework to study the 1D Hubbard model. Therefore, we will apply this scheme to calculate the response functions of the system with the aim of describing some real quasi-1D materials.
[1] J. B. Torrance, B. A. Scott, F. B. Kaufman, Solid State Commun. 17, 1369 (1975).

[2] P. W. Anderson, Phys. Rev. Lett. 64, 1839 (1990).

[3] E. H. Lieb, F. Y. Wu, Phys. Rev. Lett. 20, 1445 (1968); H. Shiba, Phys. Rev. B 6, 930 (1972); J. Carmelo, D. Baeriswyl, Phys. Rev. B 37, 7541 (1988).

[4] S. Sorella, A. Parola, M. Parrinello, E. Tosatti, Europhys. Lett. 12, 721 (1990); J. H. Xu, J. Yu, Phys. Rev. B 45, 6931 (1992); R. Preuss, A. Murahatsu, W. von der Linden, P. Dieterich, F. F. Assaad, and W. Hanke, Phys. Rev.
Lett. 73, 732 (1994).

[5] J. E. Hirsch, Phys. Rev. B 22, 5259 (1980).

[6] W. Metzner, D. Vollhardt, Phys. Rev. Lett. 59, 121 (1987).

[7] F. D. Buzatu, Mod. Phys. Lett. B 9, 1149 (1995).

[8] F. Mancini, S. Marra, H. Matsumoto, Physica C 244, 49 (1995): 250, 184 (1995): 252, 361 (1995): A. Avella, F. Mancini, H. Matsumoto, D. Villani, Physica C 282287, 1757 (1997); 282-287, 1759 (1997). 